\title{
Introduction to the Minitrack Knowledge Management Disrupted - Understanding the Impacts of Social and Mobile Media
}

\author{
Alexander Richter \\ IT University of Copenhagen \\ aric@itu.dk
}

\author{
Stefan Smolnik \\ University of Hagen \\ stefan.smolnik@,fernuni- \\ hagen.de
}

\author{
Andrea Back \\ University of St. Gallen \\ andrea.back@unisg.ch
}

Recent reports show that more and more companies adopt social media to support knowledge management (KM). These companies experience considerable changes in the way they communicate and collaborate internally once they have implemented related technologies and platforms. In addition, the challenges of the digital age make transforming underlying work practices and organizational structures more urgent. While the organizational capacity to deal with social media is maturing, we are far from embracing those changes on knowledge practices. A number of questions is to be addressed: What does the emergence of social and related mobile media mean for KM initiatives in companies? Do they have to and how do they rethink their processes and strategies? What are the timely relevant research fields for academia? Which of our established KM models do still hold, where do we need new models or frameworks? This minitrack calls for contributions that take KM one step further in the light of these contexts.

In the first year of this minitrack, after a rigorous review process, one paper (out of four) was accepted for publication in the proceedings and for presentation.

The paper is authored by Paul Beckman. He proposes modifications to the current standard approach for measuring network-based work experience and applies these modifications to a large dataset. The author's data collection and analysis show that more precise and refined measures of networkbased work experience are possible. While his domain of study is a professional sport, he argues that the proposed process can be applied to any organization in which employees work together to complete organizational tasks. This collaboration will lead to knowledge transfer from employees with greater abilities to those with lesser abilities.

We wish to thank all of the authors who submitted work for consideration in this minitrack. We also thank the dedicated reviewers for the time and effort they invested in reviewing the papers: Oliver Bendel, Sabine Berghaus, Richard Chou, Raffaele Ciriello, Xiaodong Deng, Adrian Holzer, Travis K. Huang, Michael Leyer, Florian Johannsen, Shan Liu, Mario Silic, and Nils Urbach.

We believe that the accepted paper contributes to furthering our understanding of social media's application in knowledge management. We look forward to discuss it further during our session. 